\title{
INFLUENCE OF BARK SURFACE ROUGHNESS ON TREe TrunK RADAR INSPECTION
}

\author{
J. JeŽOVÁ and S. LAMBOT \\ Université catholique de Louvain, Louvain-la-Neuve, Belgium - \\ jana.sebestov@gmail.com, sebastien.lambot@uclouvain.be \\ (J. Ježová is the Corresponding Author)
}

Ground penetrating radar; tree trunk inspection; non-destructive testing; roughness influence.

\begin{abstract}
Microwave radar testing of tree trunks is one of the ways for the trunk interior evaluation. The interpretation of the radar images can be a very complex task - among others due to the roughness of the tree bark. This paper studies the influence of a surface roughness on radar data of observed cylindrical objects, trees in particular. During our study, we did numerical simulations and laboratory measurements to compare radar data obtained by testing a cylinder with a smooth and an irregular surface. Then, several real trees with different surfaces and internal structures were tested to validate our findings. Those experiments indicate that the presence of a rough and irregular bark can significantly inhibit our ability to study the internal structure of the tree with the radar. On the other hand, if the bark is smooth, it is possible to infer the internal composition of the tree even for highly heterogeneous specimens.
\end{abstract}

Keywords: Ground penetrating radar; tree trunk inspection; nondestructive testing; roughness influence.

\section{Introduction}

Trees are a very important part of humans' lives. They are crucial for oxygen production, they are a necessary source of construction material, they have an indisputable effect on the climate and they have a significant influence on well-being in urban areas. Then, it is inevitable to pay attention to their condition. As a result of a natural degradation of wood and a human intervention to trees and their habitats, the stability of tree trunks is constantly decreasing which leads to endangering 
people and infrastructures. In order to prevent collapses of trees, it is highly important to investigate their internal structure [1]. From the macroscopic point of view, tree trunks are composed of bark, sapwood and heartwood with different mechanical properties. Within them, we can observe natural defects (knots, reaction wood, cross grain, etc.) or biological degradation (caused by fungi, insects etc.).

There are several destructive and non-destructive methods for tree trunk evaluation [2]. Core drilling, knife test or penetrometer testing are examples of the destructive ways for tree trunk inspection [3]. Gilbert et al. [4], Lin et al. [5] or Brancheriau et al. [6] used ultrasonic tomography to detect decayed wood in living tree trunks. This method is based on emitting sonic waves into the trunk and evaluating its mechanical state by the sonic waves responses. Guyot et al. [7] and Elliott et al. [8] used electrical resistance tomography (ERT) for evaluating the internal structure of tree trunks. ERT measures the subsurface distribution of electrical resistance (which is a function of humidity, density, etc.) with several electrodes following a specific geometric pattern along an investigated object. Van den Bulcke et al. [9] used X-ray tomography for analysing tree rings to measure the age of trees. Ground-penetrating radar (GPR) is being increasingly used as a non-invasive device for tree trunk interior inspection. It is based on emitting electromagnetic waves into media and capturing them after scattering on the internal structures of the studied object.

Nicolotti et al. [10] compared three non-destructive methods for the tree trunk investigation, namely, electric, ultrasonic and georadar tomography. Al Hagrey [11] also tested the same techniques for tree trunks and the root zone in order to evaluate their moisture. Butnor et al. [12] used the GPR in order to detect decays in tree trunks and provided a comparison between data obtained from gymnosperms and angiosperms. Lorenzo et al. [13] used the GPR to test a root zone of trees and tree trunks. They observed advantages of using a metal sheet to increase reflections from the other side of the tree. Fu et al. [14] provided a living tree trunk ray-based tomography using the GPR in a reflection and transmission mode. Mazurek and Łyskowki [15] tested two shielded antennas $(1.6 \mathrm{GHz}$ and $0.8 \mathrm{GHz}$ ) for a tree trunk inspection in both reflection and transmission mode in a longitudinal direction to the tree. Li et al. [16] proposed a ray-based tomography of a living tree trunk and 
compared a polar and real cross-section shape of a data visualisation. Takahashi and Aoike [17] combined the reflection and transmission mode of the GPR to distinguish heartwood and decay in logs and living tree trunks.

Tree trunk inspection using GPR is a very complex discipline for several reasons. First, living wood is a humid material with a very variable water content (which can range from about $30 \%$ to more than $200 \%$ of the weight of wood substance [18]) which is the cause of the electromagnetic waves attenuation. Second, significant heterogeneity and anisotropy of wood further complicate the interpretation of the radar data as the relative permittivity of wood depends also on a grain direction [19, 20]. Third, an irregular shape of a tree trunk does not allow for a good contact or at least a constant distance between a radar antenna and the tree trunk surface. Having various distances between the surface and the antenna leads to irregular surface reflections in radar images which are not straightforward to filter out. And last, but not least, in order to inspect tree trunks, it is essential to keep a good contact of the antenna with bark for better impedance matching. This is not always possible due to the bark roughness.

The influence of the surface roughness on radar data was already very well described by Pinel et al. [21] who studied wave scattering from multilayered random rough surfaces for road applications. Tosti et al. [22] dealt with the roughness of a railway ballast during evaluation of its dielectric properties with the GPR. Ardekani et al. [23] studied scattering and attenuation of radar signal due to a vegetation cover. Lambot et al. [24] and Jonard et al. [25] observed the soil roughness influence on the monostatic GPR signal inversion to retrieve surface moisture.

The objective of this paper is to see the influence of the tree trunks rough surface on GPR images. In that respect, we simulated two cylindrical configurations with a smooth and rough surface using a finite difference time domain simulator, namely, gprMax2D [26, 27]. To check the validity of the numerical simulations, two laboratory measurements on a corresponding cylindrical model were done. The laboratory model contained also a smooth and a rough surface. Finally, four radar acquisitions on real trees with various surfaces and internal structures were carried out. Two trees with a rough bark and two trees with a smooth bark were chosen. Both pairs of trees consisted of a relatively 
healthy tree and a tree with a visible cavity. Data were processed using two filtering methods: free-space response subtraction and the average background removal. For more intuitive readability of the GPR images, the cartesian radargrams were projected to the polar coordinates.

\section{Numerical simulations}

The first step in understanding the effect of the surface roughness on our measurements was to simulate a cylindrical structure with a smooth and with a rough surface. In our previous experiments [28, 29], we studied reflections curves occurring in simulated and measured radargrams of a cylindrical laboratory model. All those experiments were done for a smooth surface. To see the influence of a rough surface, the same configuration with a rough surface was simulated. The configuration had a similar shape as a laboratory cylindrical model which is filled with sand. The rough surface was designed to have a random thickness (up to $70 \mathrm{~mm}$ ) while the internal parts of the model remained the same (dry sand with air). The rough surface was created by generating 1000 random points in a known annular area with outer radius $4 \mathrm{~cm}$ larger than the current model. The generated points were used as centres of a series of filled circles (with a radius of $3 \mathrm{~cm}$ ). The circles randomly overlapped each other and created a filled area with a rough edge. Figure 1 shows a configuration with a smooth surface (a) and with a stochastic rough surface (b). The relative permittivity of the sand was set to 3 and the relative permittivity of the generated surface was set to 2 to be slightly different than sand. The numerical simulations were done using the open source software gprMax2D [26, 27] using the Finite-Difference Time-Domain (FDTD) method [30] and which is specifically dedicated to GPR applications. The operating source was a Ricker wavelet with a centre frequency of $f_{c}=900 \mathrm{MHz}$. The spatial resolution of the numerical model geometry in the $x$ and $y$ directions was $2,5 \mathrm{~mm}$.

Figure 2 shows the simulated radargrams of the smooth configuration (left) and the configuration with the random rough surface (right) for both choices of data filtering (free-space response subtraction and average background removal). In all images, we can see the surface reflection (at about $1.5 \mathrm{~ns}$ ), the reflection from the opposite side of the model (at about $10 \mathrm{~ns})$, the reflection curves originating from internal inhomogeneities 


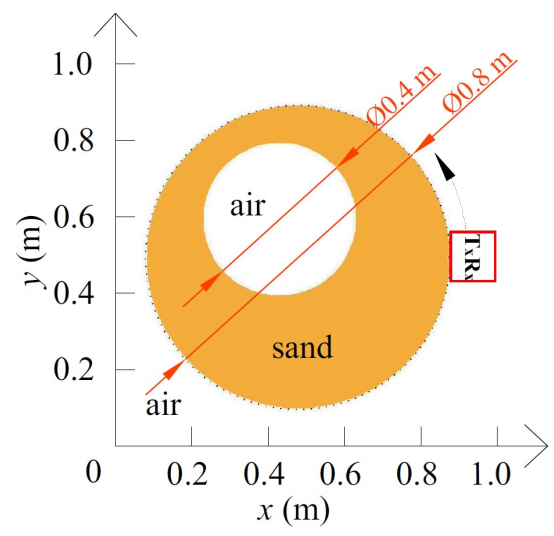

(a)

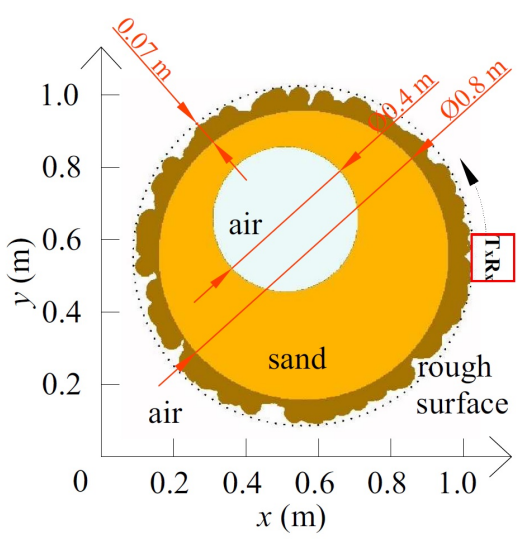

(b)

Figure 1: Configurations of the tree trunk model for numerical simulations in gprMax2D: a) With a smooth surface, b) with a rough surface with the tickness up to $7 \mathrm{~cm}$.

(two sinusoidal shapes at about 3-7 ns corresponding to the closest and the furthest point on the void to the receiver) and the total internal reflection (TIR) at about 14 ns (explained by Ježová et al. [28, 29]).

Figure 2a (smooth surface, free-space response subtraction) provides very clear reflection curves described above without any particular noise (other minor reflections). The reflections of the opposite side of the model (at $10 \mathrm{~ns}$ ) and the TIR are stronger at the positions of 0.5-1.2 $\mathrm{m}$ when the antenna is close to the internal void. Then, at the propagation time of 7-14 ns we can see cross-shaped reflections at the positions of 1.7-2.5 $\mathrm{m}$ when the antenna is far from the internal void. Both cases occur due to the symmetrical geometry regarding the antenna position and the internal void which can cause additional reflections. We already described this phenomenon in our previous study [31].

In Figure $2 b$ (rough surface, free-space response subtraction), many reflections in the background are present. This fact, however, does not prevent the visibility of the internal void reflections and the TIR, but it makes other reflections less visible (e.g., the opposite side reflection at $10 \mathrm{~ns})$. Just below the surface reflection, we can observe a reflection originating from the interface between the rough surface and the cylinder. In the image, the opposite side of the observed model is not represented by a line, but we can see it through a series of irregular reflections 
at about $10 \mathrm{~ns}$. It is worth mentioning that we cannot see any additional reflections caused by the symmetry of the model-hole-antenna configuration because this phenomenon was significantly suppressed by the rough surface.

In Figure 2c (smooth surface, average background subtraction), the reflections pointing at the internal inhomogeneities, opposite side reflection and TIR are well visible. Nevertheless, the surface reflection was almost fully eliminated by the average background removal as it is constant. Furthermore, a constant line at about 3 ns now appeared in the radargram as a result of the filter. In the radar data, we can see a strong reflection at $3 \mathrm{~ns}$ in positions of $0.9-1 \mathrm{~m}$ as a part of the sinusoidal reflection of the internal void. Its values affected the average value which was subtracted from the whole image at this time. This may cause problems during a tomography of the medium as it displays a non-existing external contour of the model. Also in this case, we can observe a stronger opposite side reflection curve and the TIR as well as the cross-shaped reflections caused by the symmetrical geometry.

In Figure 2d (rough surface, average background subtraction), we can again observe many minor reflections caused by the rough surface, especially at the closer edge of the configuration (at about 1-2 ns) and its opposite side (at about $10 \mathrm{~ns}$ ). The strong constant reflections appearing in Figure 2b (surface reflection and TIR) were weakened but generally, the images are very similar for both choices of the filtering. Also in this case, a constant line at $3 \mathrm{~ns}$ appeared as a result of the use of the average background removal.

To better visualize of the GPR images, a polar representation of them was made. To keep the surface reflection of the configurations, the images processed with the free-space response subtraction were chosen. To project into the polar representation of the radargram, the GPR image was cut in the middle part corresponding to approximately $4.6 \mathrm{~ns}$ (according to its $\varepsilon_{r}=3$ and the geometric radius $r=0.4 \mathrm{~m}$ ) from the source reflection ( $1.5 \mathrm{~ns})$. Therefore, the image was split at the propagation time of 6.1 ns. Figure 3 a shows the polar representation of the simulated radargram of the smooth case. We can see very well the external and internal contours of the configuration without any additional reflection. The image is very clear and corresponds to the simulated cylinder also with its size. Figure $3 b$ shows the polar representation of the 


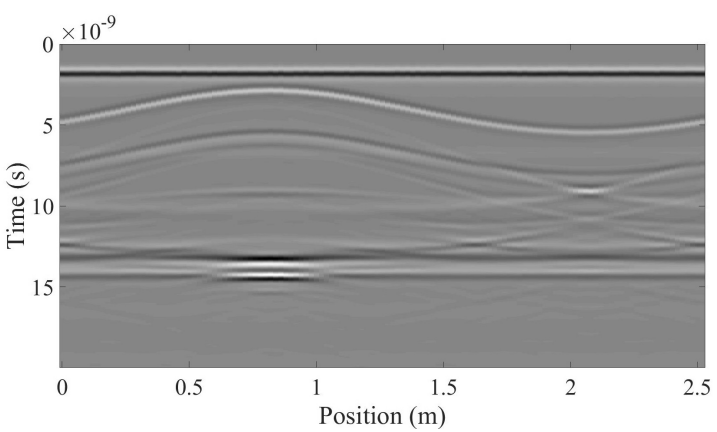

(a)

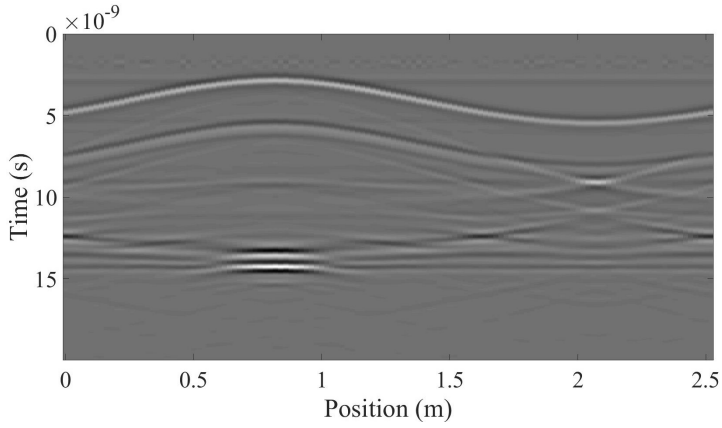

(c)

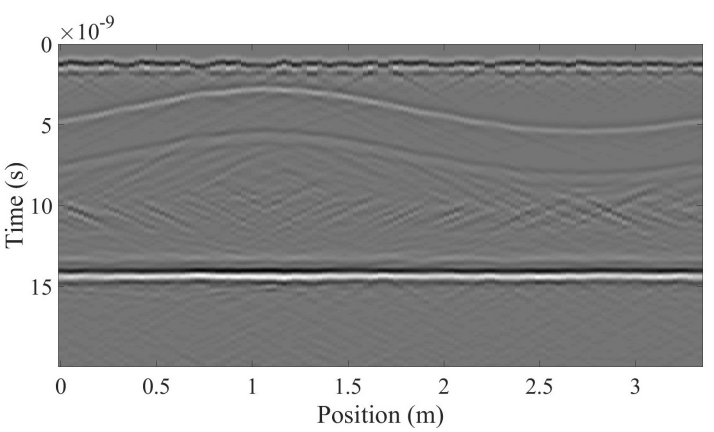

(b)

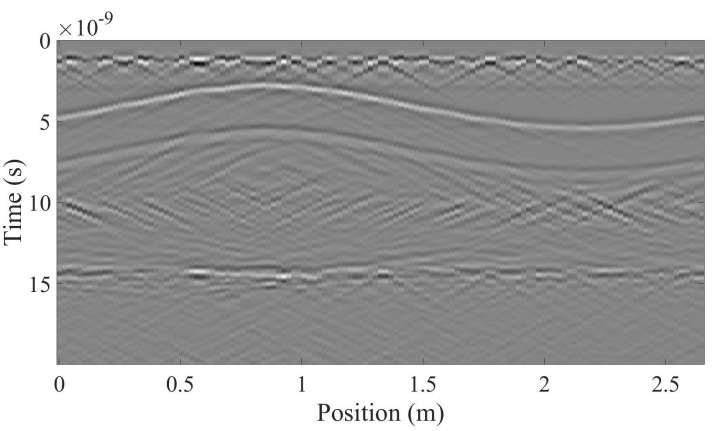

(d)

Figure 2: Numerical simulations using gprMax2D of a laboratory tree model with a smooth surface (left) and with a rough surface (right). The radar data were processed: a-b) with a free-space response subtraction, c-d) with an average background subtraction.

configuration containing the random rough surface. We can observe the very rough surface of the model edge and the slightly smoother surface of the circle which follows. The internal void is very well displayed despite the rough surface. In both images, a small reflection (coordinates [0.6, 0.6] in Figures $3 a$ and 3b) appeared as an artifact of the projection. This indicates, that such a visualization is only illustrative, as we calculated the model centre assuming homogeneous permittivity of $\varepsilon_{r}=3$, which is violated by the presence of the internal void.

\section{Laboratory measurements}

The next step of the rough surfaces evaluation was done in laboratory conditions. In particular, we performed two radar measurements of a laboratory cylindrical model made from two tubes (paper and PVC) and 


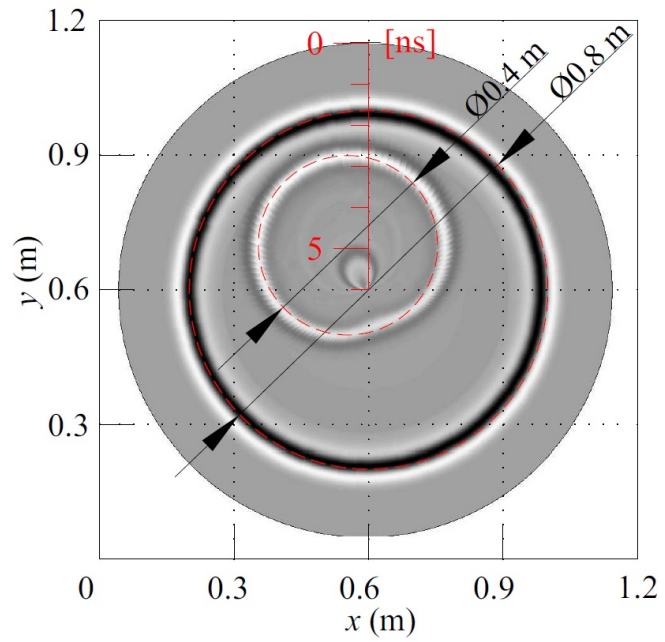

(a)

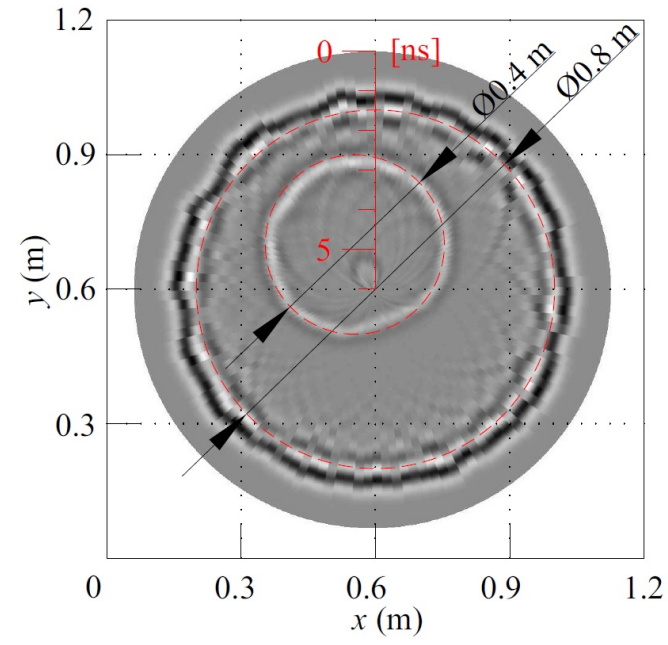

(b)

Figure 3: Polar representations of the numerical simulations with gprMax2d of the configuration with: a) Smooth surface, b) rough surface. The simulated data were processed with the free-space response subtraction.

sand (as a filling material). Analogically to the numerical simulations, the cylindrical model had a smooth surface for the first measurement while for the second, an irregular inhomogeneous structure made of paper and polystyrene was installed on the external surface of the larger tube to emulate the shape of the tree bark (see Figure 4). Both measurements were carried out with a lightweight radar system including a dielectric-coupled TEM horn antenna, a micro vector network analyser (Planar R54, Copper Mountain Technologies, Indianapolis, USA, [32]), an Intel computer Stick, a battery and a webcam for remote positioning. The antenna was filled with paraffin wax $\left(\varepsilon_{r}=2.2\right)$ in order to better couple the antenna impedance with the investigated medium (dry sand with $\varepsilon_{r}=3$ ). This radar system (see Figure 5) was described in detail in [32]. In order to get the return loss of the antenna $H_{i}$, it was calibrated against a copper sheet $(3 \times 3 \mathrm{~m})$ using the antenna model of Lambot et al. ( [33]). For the calibration, a VNA (ZNB8, Rohde \& Schwarz, Munich, Germany) was used to feed the antenna. The VNA was calibrated following Open-Short-Match reference calibration kit standards. The operating frequency was set to 0.5-3.5 GHz with a frequency step of $2 \mathrm{MHz}$.

In order to validate the observations made about different approaches 


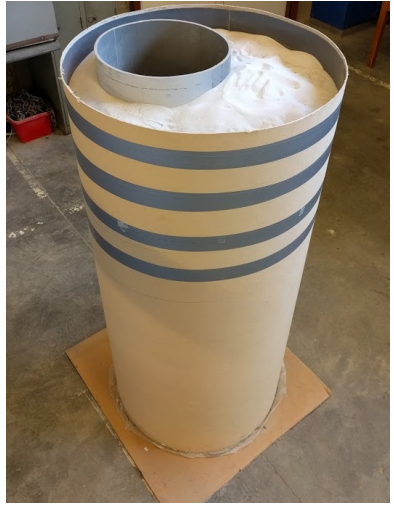

(a)

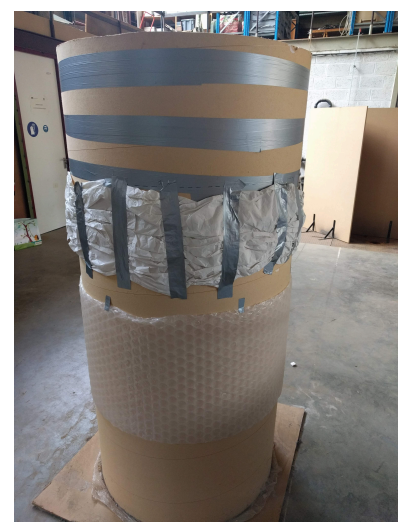

(b)

Figure 4: Laboratory tree trunk model: a) with a smooth surface, b) with a bark.

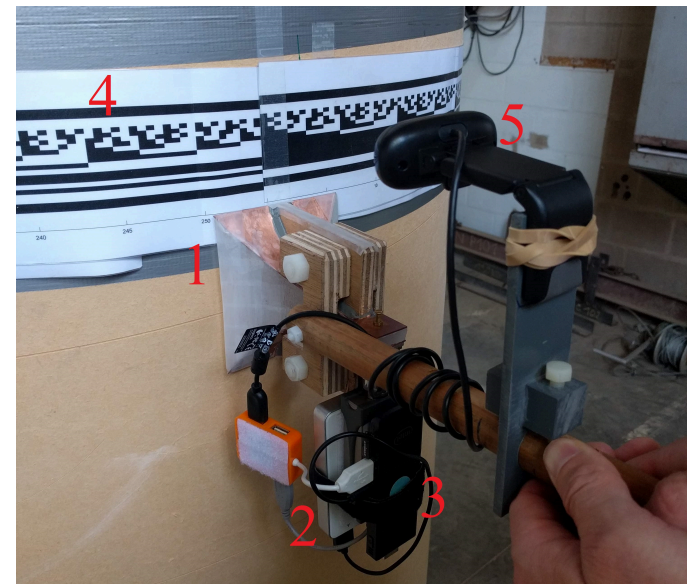

Figure 5: The mini radar system set up for the measurement of the laboratory cylindrical model with a smooth surface. 1) dielectric TEM horn antenna, 2) battery with VNA, 3) Intel computer, 4) barcode ruler, 5) webcam for remote positioning.

to background removal in the previous section, two different types of filtering was used again, namely, the free-space response subtraction (Figures 6a and 6b) and the average background subtraction (Figures 6c and 6d). Unsurprisingly, the laboratory images have different appearance than the simulated ones, especially those treated with free-space response subtraction where the antenna effects were removed. Both images with this filter contain periodically repeating multiple reflections between the antenna and the medium. In the simulated images, we used a point source dipole for emitting the signal, however, in the 
laboratory conditions a real antenna was used. By free-space response subtraction, we eliminated the internal reflections in the antenna, but not the reflections originating from its contact with the medium.

Both cases (smooth and rough) have very similar appearance and we can observe the surface reflection (at about $1.2 \mathrm{~ns}$ ) and the internal void reflection (sinusoidal shape at about 2-5 ns). We can also see the strongest parts of the TIR (14 ns, 0.5-1.2 m) and the cross-shaped reflection (0.7-14 ns, 1.7-2.5 m) which we were able to see much better in the simulation. The remaining reflections are not recognizable very well as they have been obfuscated by the multiple reflections from various surfaces that have not been included in the simulation.

The second set of GPR images (using the average background subtraction) are, compared to free-space response subtraction, very well readable. We can immediately see that they describe a cylinder with the same internal structure but different surfaces. Figure $6 \mathrm{c}$ shows the GPR image of the smooth laboratory cylinder. As for the simulated case, the surface reflection was practically eliminated by the average background subtraction because the antenna was in contact with the surface and, therefore, the distance was constant and so, easily eliminated by this filter. Nevertheless, we can see very well the sinusoidal reflection from the internal void closest point (at about 2-5 ns) and the furthest point (about $2.6 \mathrm{~ns}$ further) as two parallel curves. We can also see a relatively constant reflection curve at about $10 \mathrm{~ns}$ which points at the opposite side of the cylinder. At $14 \mathrm{~ns}$, we can see the TIR which is not visible so well as in the simulated images, but it is recognizable. Same as during the simulations, the curves are thicker at the positions 0.5-1.2 m. The cross-shaped reflection (0.7-14 ns, $1.7-2.5 \mathrm{~m}$ ) is again well visible. At the position of $1.3 \mathrm{~m}$ and the time of about $1.2 \mathrm{~ns}$, we can see a very strong hyperbolic reflection corresponding to a metallic structural wire hidden in the laboratory cylinder.

Figure $6 \mathrm{~d}$ shows the GPR image of the laboratory cylinder with the rough surface. We can see more or less the same reflections as in the previous case with the smooth surface (Figure 6c). Also here we can see the opposite side reflection curve which is bolder at the positions 0.5-1.2 $\mathrm{m}$ and the cross-shaped reflection (0.7-14 $\mathrm{ns}, 1.7-2.5 \mathrm{~m})$. This illustrates the fact, that the bark laboratory emulation is less rough than the one used in the numerical simulations. Nevertheless, we can 


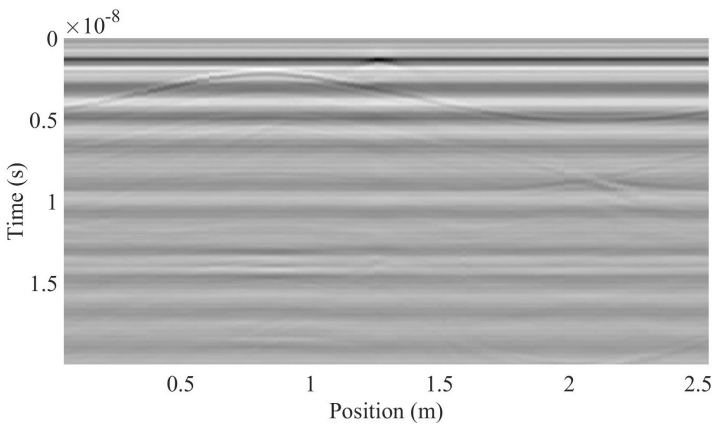

(a)

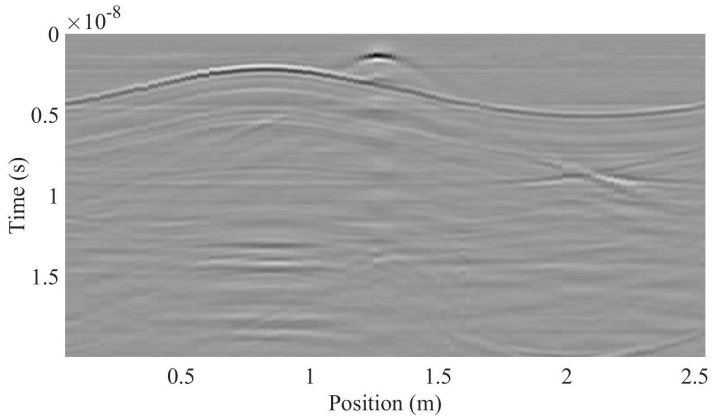

(c)

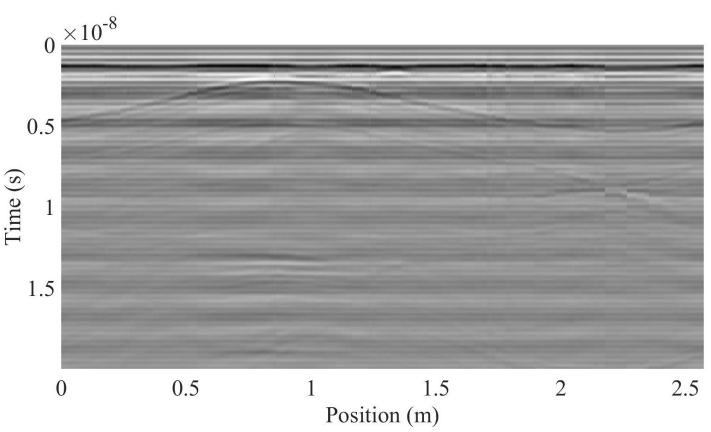

(b)

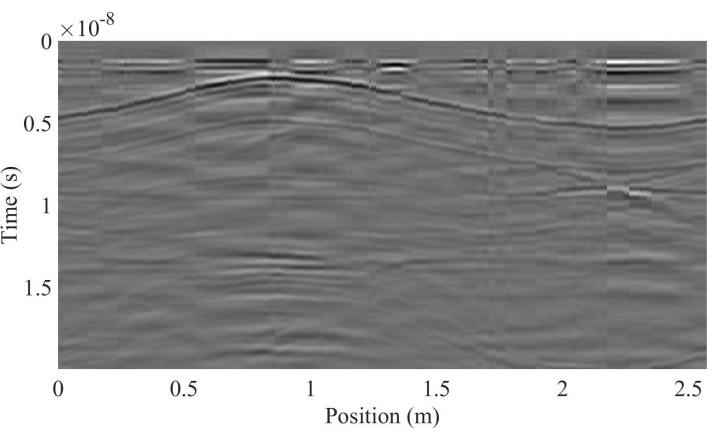

(d)

Figure 6: A laboratory tree trunk model measuremen with a smooth surface (left) and with a bark (right). The radar data were processed: a-b) with a free-space response subtraction, c-d) with an average background subtraction.

observe certain differences. First, the reflection corresponding to the surface of the cylinder was not eliminated by the average background removal, because the distance between the antenna and the surface was not constant due to the irregular (rough) artificial surface. We cannot, however, see the direct reflections corresponding to the artificial bark created on the laboratory model surface as paper and polystyrene have similar $\varepsilon_{r}$ to the air. Second, the surface-antenna interaction caused the same multiples as we could see in Figures $6 \mathrm{a}$ and $6 \mathrm{~b}$, but because of the rough surface, it is not distributed constantly in direction $x$ (position change). Hence, the average background subtraction could not eliminate it. Therefore, there is a lot of noise which makes the image more difficult to interpret. Finally, we can see only hints of the metallic wire reflection due to the noise in the radargram.

We represented the laboratory radargrams in the polar coordinates in the same way as we did for the simulated radar images. In 


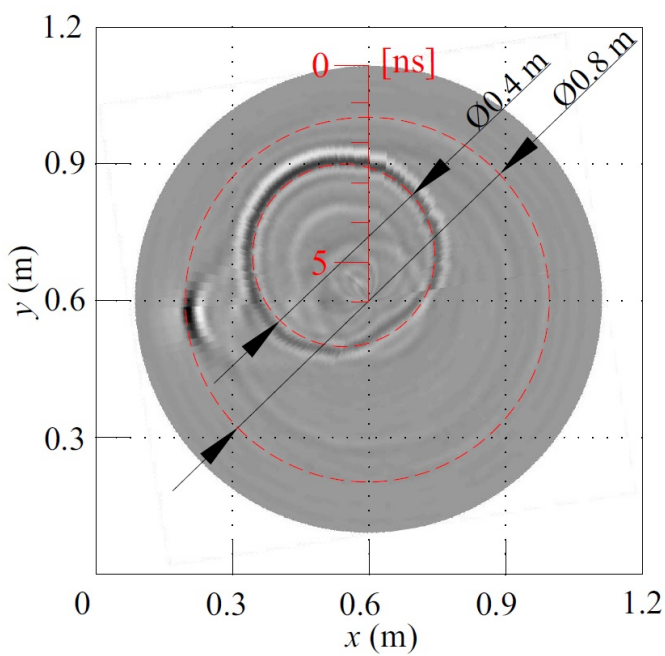

(a)

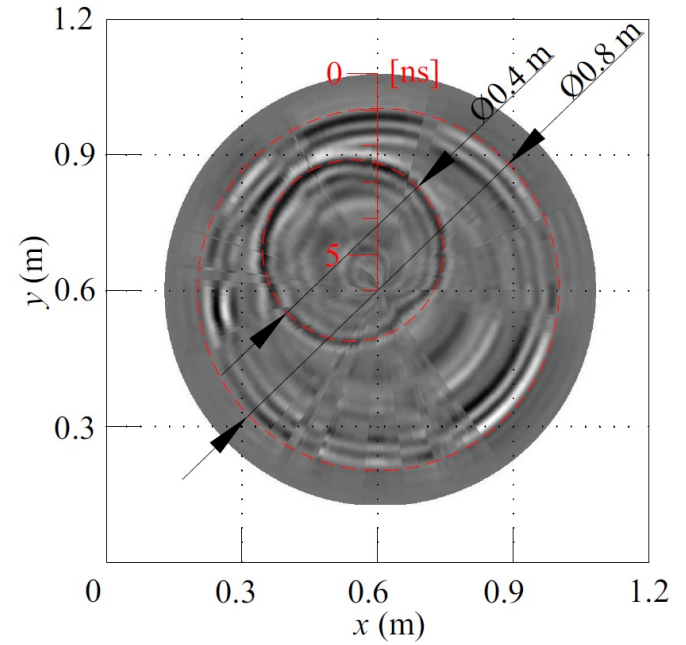

(b)

Figure 7: Polar representations of the simulated data of the configuration with: a) Smooth surface, b) rough surface. The simulated data were processed by the average background removal.

contrast to the previous section, the second filtering method (average background removal) exhibited better readability of the radargrams than the free-space response subtraction due to missing multiple reflections. Figure 7a displays the smooth cylindrical model radargram with two outstanding reflections pointing at the internal void at the position $(0.5$, $0.7)$ and the metallic wire at the position $(0.2,0.6)$. Due to the average background subtraction, the contour of the model surface is not so well visible which makes it difficult to read comfortably the image without the red contour. Figure $7 \mathrm{~b}$ shows the polar representation of the radargram of the cylinder with the rough surface emulation. In this case, the model external border is well visible as well as the internal void. The image is, nevertheless, quite noisy.

\section{Real tree trunk measurements}

To see the real influence of a bark and of an internal structure on the radar data acquisition, we performed several measurements on real tree trunks. For the experiments, we chose four trees with different external and internal properties. All measurements were performed 


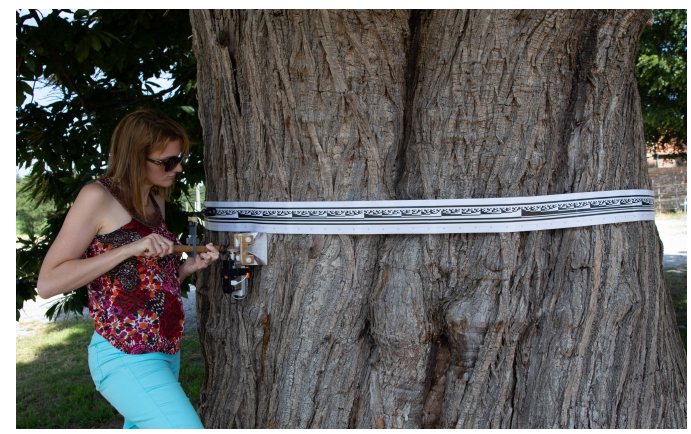

Figure 8: A living sweet chestnut with a rough bark in Waterloo (Belgium).

using the same lightweight radar system with a dielectric-coupled TEM horn antenna as for the laboratory measurements. The frequency range was set again to 0.5-3.5 GHz. All measurements were done in near-field conditions where the antenna was in contact with the bark. For all radar images, we applied the average background removal, as it showed better performance during the laboratory tests and an exponential gain function.

\subsection{Living tree with a rough bark (Waterloo - Chestnut)}

The first case study was a living tree (sweet chestnut, Castanea Sativa) in the Castle-Farm of Hougoumont, Waterloo, Belgium, with a very rough bark (see Figure 8). The tree is very old as it was present during the Battle of Waterloo in 1815 (the tree is situated in the battle site). This tree had about $6 \mathrm{~m}$ in circumference (almost $2 \mathrm{~m}$ in diameter). The radar data acquisition was not very easy due to the exceptional roughness of the bark and also the very irregular shape of the tree trunk cross-section. Hence, the antenna was never fully in contact with the surface of the tree trunk. Furthermore, only $5 \mathrm{~m}$ of its circumference was inspected because of poor accessibility (bushes around the trunk).

Figure 9 displays a radar image of that tree. The polar representation shows the part of the tree trunk which was investigated. For the polar representation, the $\varepsilon_{r}$ of the medium was estimated to be 9 (as a living old tree) leading to $20 \mathrm{~ns}$ as the centre of the trunk because the diameter of the tree was about $2 \mathrm{~m}$. We can observe very clearly the surface reflection (red curve at about $1.2 \mathrm{~ns}$ ) which is very irregular. Another reflection (green curve) is visible at about $2.5 \mathrm{~ns}$, and it has the same shape as the 


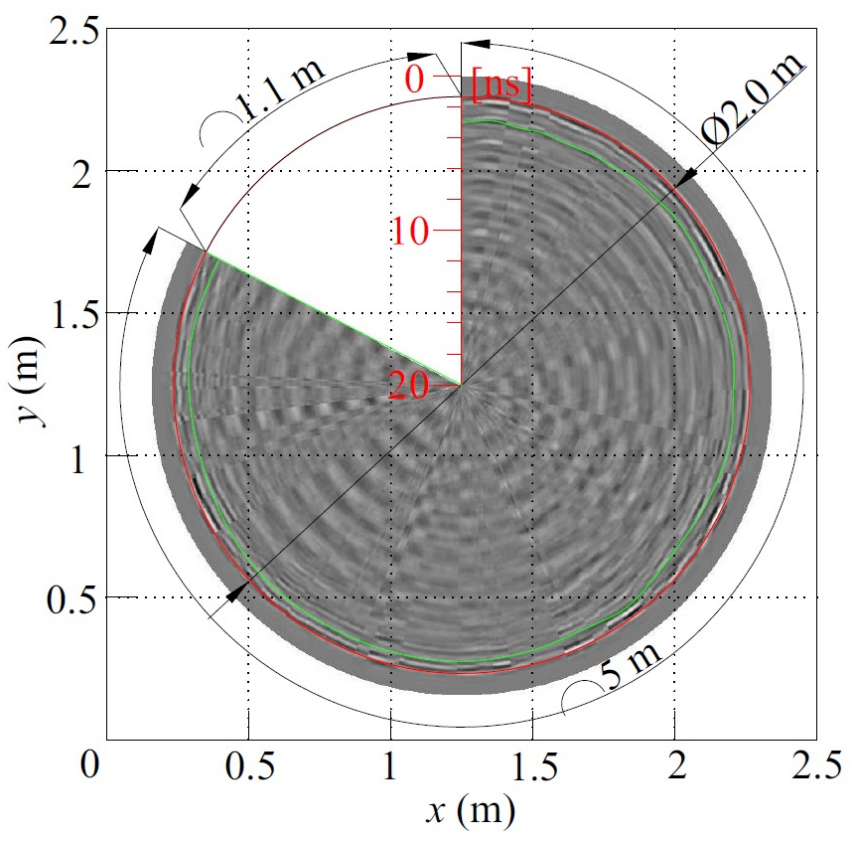

Figure 9: A polar representation of the radar image of a living chestnut with a bark (in Waterloo) up to 20 ns which is the estimated centre of the trunk (for $\varepsilon_{r}=9$ ). Surface reflection is highlighted with a red curve, its first multiple reflection is highlighted with a green curve.

surface reflection. This means, that the reflection at $2.5 \mathrm{~ns}$ is a multiple reflection of the surface which repeats again, but much more feebly also at about $4 \mathrm{~ns}$ in the radargram (and the next ones would be more visible if we used higher gain function). The rest of the image is very noisy despite the applied filter. Therefore, we can assume, that nearly all waves were reflected from the bark or absorbed in the relatively humid sapwood.

\subsection{Living tree with a rough bark and a cavity (Sohier - Oak)}

Another case study was the inspection of a living oak (Quercus robur) with a rough bark and with a significant cavity in the trunk (see Figure 10). This tree was situated in the countryside in Sohier, Belgium. The tree trunk cross-section was more regular than the one of the previous tree and the texture bark was slightly finer. The circumference of the tree was about $4 \mathrm{~m}$ (giving the diameter of about $1.3 \mathrm{~m}$ ). Its bark was partly missing and through one side we could see an open internal cavity of a very irregular shape $(20-40 \mathrm{~cm}$ in diameter). 


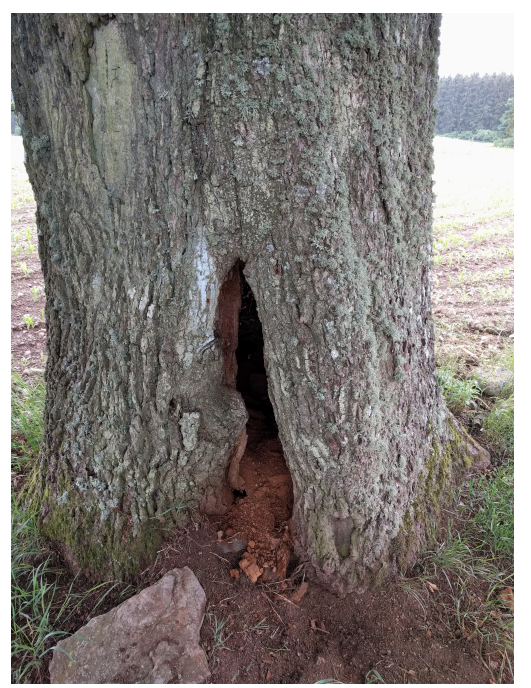

Figure 10: A living oak with an internal cavity in Sohier (Belgium). Missing bark and the opening to the internal cavity.

Figure 11 shows a radar image in polar coordinates obtained by the radar inspection of the oak. We present only the part of the radargram with outstanding reflection curves (about $43 \%$ of the circumference) because the radar data were affected by very strong reflections at the positions corresponding to a cavity or a missing bark. Same as for the previous tree, we evaluated the $\varepsilon_{r}$ to 9 and so, we determined the centre of the trunk to be at the propagation time of $13 \mathrm{~ns}$ (for a diameter of about $1.3 \mathrm{~m}$ ). We can see the surface reflection at about $1.2 \mathrm{~ns}$ (red curve) and also its multiple at about $2.5 \mathrm{~ns}$ (blue curve). This radar image is very noisy due to the rough surface and very heterogeneous medium, but we can observe certain reflection curves at 5-6 ns (green curve). This reflection points at the internal irregularity (probably the cavity) in the trunk. In this example, we can see an importance of the electromagnetic properties evaluation. We set the $\varepsilon_{r}$ as a homogeneous value for the whole tree trunk, nevertheless, the $\varepsilon_{r}$ in the cavity was 1 . Therefore, the size of the cavity appears larger in the polar representation $(\approx 0.9 \mathrm{~m})$ than in reality $(\approx 0.4 \mathrm{~m})$.

\subsection{Living tree with a smooth bark (Louvain-la-Neuve - Beech)}

The third case study was a radar inspection of a living European beech (Fagus sulvatica) in a forest in Louvain-la-Neuve, Belgium, with 


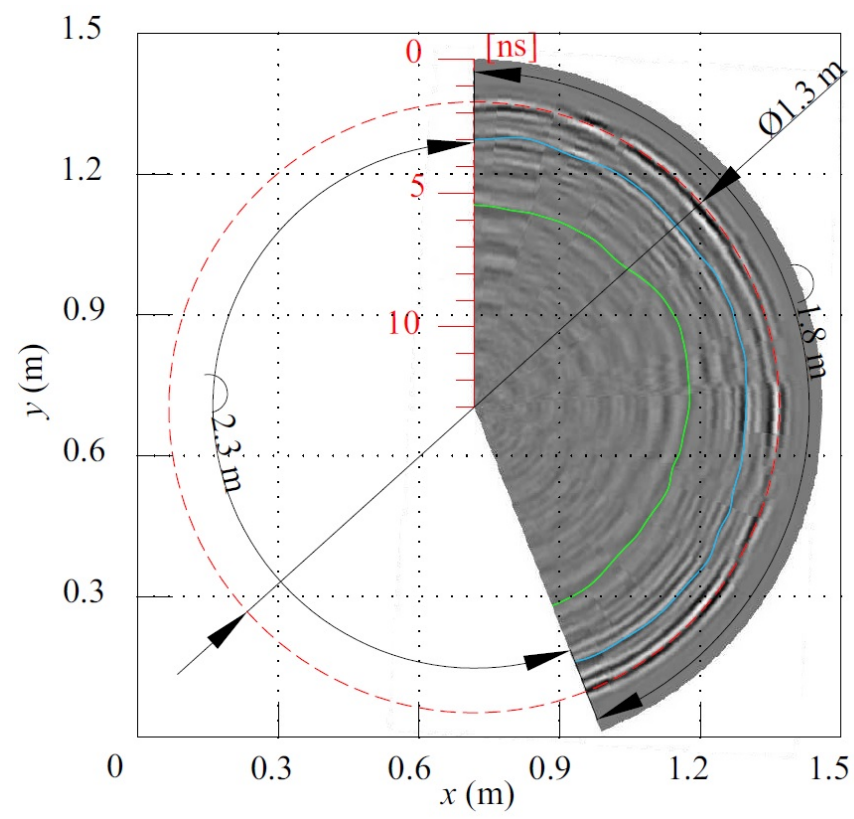

Figure 11: A polar representation of a radar image of a living oak with an internal cavity (in Sohier) until 13 ns which is the estimated centre of the trunk (for $\varepsilon_{r}=9$ ). Red curve points at the surface reflection, blue curve shows its multiple and green cruve highlights the internal cavity.

a relatively smooth bark (see Figure 12). This tree had a circumference of about $3 \mathrm{~m}$ (almost $1 \mathrm{~m}$ in diameter), and it had a relatively regular cross-section (circular shape). It was therefore relatively easy to keep the contact between the antenna and the tree trunk surface without almost any air gaps.

In Figure 13, the radar image of the living beech tree is displayed in polar coordinates. We estimated the centre of the trunk at about $11 \mathrm{~ns}$ (for a relatively healthy living tree trunk, $\varepsilon_{r}=12$ ), knowing the tree trunk circumference $3 \mathrm{~m}$ and so, it diameter almost $1 \mathrm{~m}$. Because the distance between the antenna and the surface was almost constant, it was easy to reduce multiple reflections which were constant too. The image then appears less noisy than the previous cases. In Figure 13, the surface reflection at $1.2 \mathrm{~ns}$ is highlighted by a red curve. The curve is very clear and quite smooth. Afterwards, closer to the centre, we can observe other reflections with a different shape than the surface contour (blue and green curves). Those reflections correspond to certain interfaces inside the tree trunk. Changes of electromagnetic properties may correspond to 


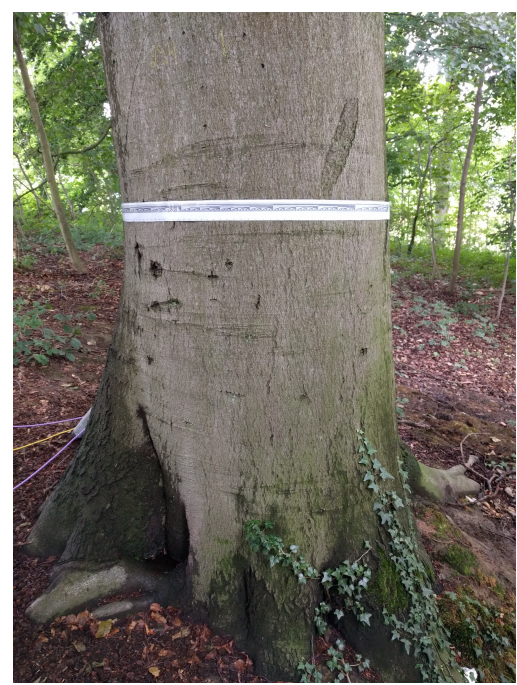

Figure 12: A living beech tree in Louvain-la-Neuve (Belgium).

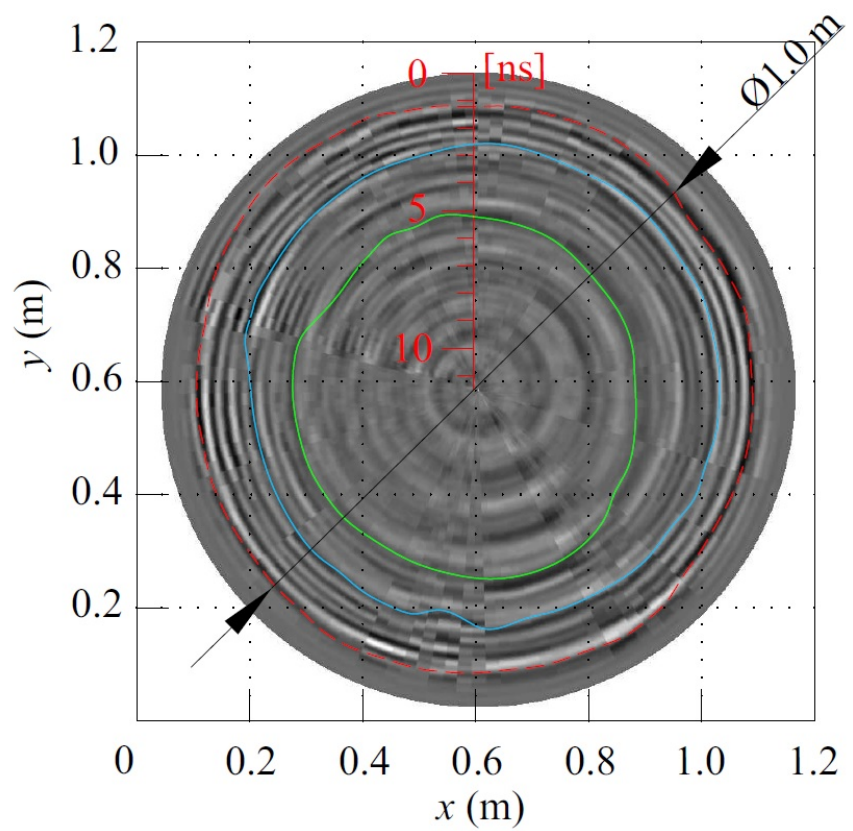

Figure 13: A polar representation of the living beech (in Louvain-la-Neuve) radar image until $11.5 \mathrm{~ns}$ which is the estimated centre of the trunk (for $\varepsilon_{r}=12$ ).

changes of humidity or density of wood. It will then point at heartwood or decayed wood. Because we can see two layers in the trunk, we assume to have detected decayed wood in different stages of rot. 


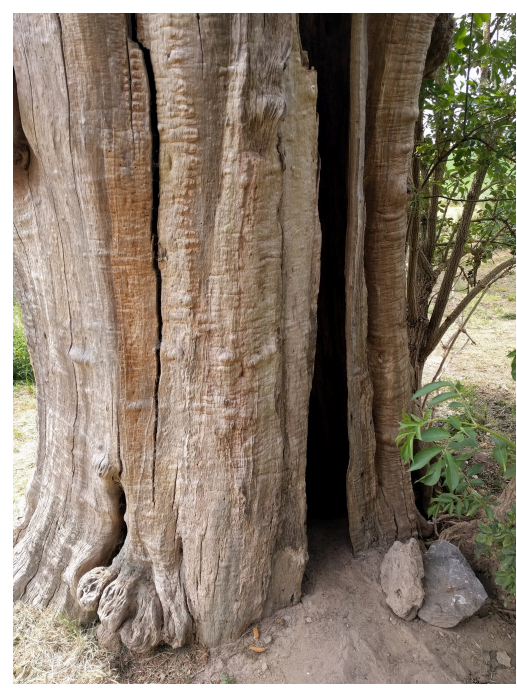

Figure 14: A dead sweet chestnut in Waterloo (Belgium).

\subsection{Dead tree without bark (Waterloo - Chestnut)}

The last case study was a radar inspection of a dead sweet chestnut (Castanea Sativa) in the Castle-Farm of Hougoumont, Waterloo, Belgium (see Figure 14). This tree was visibly dead. It was already without its bark and inside the tree trunk. We could see a large irregular cavity. The cross-section of the trunk was relatively regular and the trunk surface was very smooth. The circumference of the tree trunk was about $4.5 \mathrm{~m}$ which gave us its diameter of about $1.4 \mathrm{~m}$. The inspection was not done around the whole trunk because of a difficult accessibility (bushes).

In Figure 15, we can see a radar image in polar coordinates obtained by the radar inspection of the dead sweet chestnut. We estimated $\varepsilon_{r}$ for this tree to 5, as the tree was dead and dry, and moreover, it contained a large cavity. Then, the centre of the trunk was calculated to approximately $11 \mathrm{~ns}$ (knowing the diameter about $1.4 \mathrm{~m}$ ). The image shows the part of the trunk which was investigated. The wedge in the lower part of the image corresponds to the opening to the cavity in the tree trunk. In this part, only multiple reflections are visible, as the antenna was in free space. Nevertheless, the rest of the image shows the data obtained by the radar measurement when the antenna was in contact with the bark. The surface reflection is, again, highlighted by a red curve. It is quite regular and by its shapes, it corresponds to the real trunk surface shape. Then, we can see several reflections (blue, green 


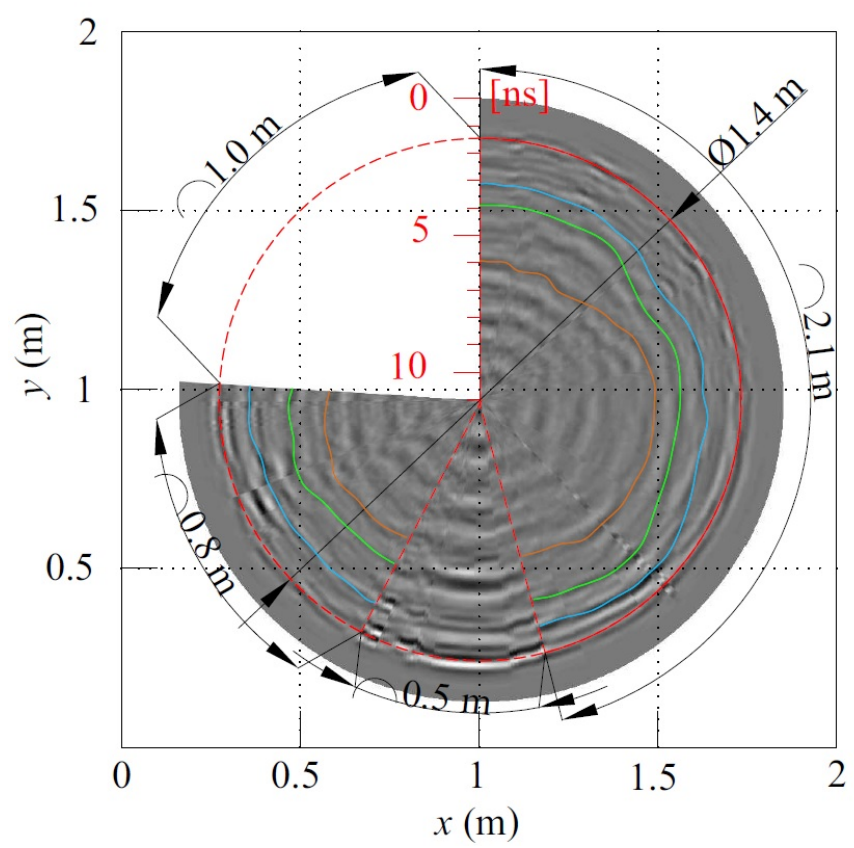

Figure 15: Polar representation of the dead sweet chestnut (in Waterloo) radar image until $11 \mathrm{~ns}$ which is the estimated centre of the trunk (for $\varepsilon_{r}=5$ ).

and orange) pointing at the very complex internal structure of the tree trunk.

\section{Summary and Conclusions}

In this paper, we studied the influence of the surface roughness on the appearance of radar images, specifically for tree trunk radar inspection. We performed simulations comparing a cylindrical model with a smooth and a rough surface. To check the correctness of the simulation, we performed measurements on a laboratory cylindrical model with a smooth and a rough surface corresponding to the simulated configuration. The radar data were treated using two different filters, namely, free-space response subtraction and average background subtraction.

In both cases, the rough surface made the GPR images noisy and less readable. It did not prevent detection of the large inhomogeneities in the observed model, but it made observation of the small objects (e.g, the metal wire hidden in the laboratory cylinder) very complicated. 
Furthermore, the presence of the irregular surface practically eliminated the phenomenon originating from a symmetrical antenna-cylinder geometry.

For the two cases (numerical simulations and laboratory measurements), a different type of filtering turned out to be more suitable. The free-space response subtraction, which worked perfectly for the simulated images, did not provide a good filtering for the real measurements, as it did not eliminate the reflections originating from the interaction between the antenna and surfaces. Therefore, the average background subtraction was used for the measurements with the real antenna even though it removed the constant surface reflection which was important for expressing the radar image in a realistic way.

Despite the fact that the mini-radar system provided very good radar images of the laboratory cylindrical model, we can observe certain limitations for a tree trunk inspection. We performed radar measurements of four types of trees, specifically, two trees with a rough bark, two trees with a smooth bark. Both pairs consisting of a relatively healthy tree and a tree with a cavity. The radar data show the difficulty of a satisfactory data acquisition of trees with a rough bark. The signal was significantly reflected which prevented detecting internal interfaces in living wood, which is very humid and causes signal attenuation. On the other hand, the radar data obtained by the measurement of the trees with a smooth bark showed relatively clearly the internal structure of the trunks despite the living wood humidity.

We demonstrated that the major limitation of the tree trunk inspection with the GPR is most likely the high degree of irregularity of the bark. Additionally, to improve the tree radar data, we suggest a usage of dielectric antennas with similar electromagnetic properties of wood. To avoid having air gaps between the antenna and the tree trunk surface, a flexible material may be considered as a dielectric for future antennas.

\section{Acknowledgment}

The authors would like to acknowledge the support of the Fonds de la Recherche Scientifique (FNRS), Belgium, through the SENSWOOD project (Convention $\mathrm{n}^{\circ}$ 19526260). We would like to thank the staff of the Castle-Farm of Hougoumont, Waterloo, Belgium, who allowed us to 
perform the measurements. This research was also carried out within the framework of EU funded COST Action TU1208 "Civil Engineering Applications of Ground Penetrating Radar".

\section{References}

[1] F. Kollmann and W. Cote, Jr., Principles of Wood Science and Technology I, Solid Wood. Berlin Heidelberg New York: Library of Congress Springer-Verlag, 1968, vol. Catalog Card Number 67-29 614.

[2] P. Niemz and D. Mannes, "Non-destructive testing of wood and wood-based materials," Journal of Cultural Heritage, vol. 13, no. 3 SUPPL., pp. S26-S34, September 2012.

[3] M. Larjavaara and H. C. Muller-Landau, "Comparison of decay classification, knife test, and two penetrometers for estimating wood density of coarse woody debris," Canadian Journal of Forest Research, vol. 40, no. 12, pp. 2313-2321, November 2010.

[4] G. S. Gilbert, J. O. Ballesteros, C. A. Barrios-Rodriguez, E. F. Bonadies, M. L. Cedeño Sánchez, N. J. Fossatti-Caballero, M. M. Trejos-Rodríguez, J. M. Pérez-Suñiga, K. S. Holub-Young, L. A. W. Henn, J. B. Thompson, C. G. García-López, A. C. Romo, D. C. Johnston, P. P. Barrick, F. A. Jordan, S. Hershcovich, N. Russo, J. D. Sánchez, J. P. Fábrega, R. Lumpkin, H. A. McWilliams, K. N. Chester, A. C. Burgos, E. B. Wong, J. H. Diab, S. A. Renteria, J. T. Harrower, D. A. Hooton, T. C. Glenn, B. C. Faircloth, and S. P. Hubbell, "Use of sonic tomography to detect and quantify wood decay in living trees," Applications in Plant Sciences, vol. 4, no. 12, p. 1600060, December 2016.

[5] C.-J. Lin, Y.-C. Kao, T.-T. Lin, M.-J. Tsai, S.-Y. Wang, L.-D. Lin, Y.-N. Wang, and M.-H. Chan, "Application of an ultrasonic tomographic technique for detecting defects in standing trees," International Biodeterioration \& Biodegradation, vol. 62, no. 4, pp. 434 - 441, December 2008. 
[6] L. Brancheriau, A. Ghodrati, P. Gallet, P. Thaunay, and P. Lasaygues, "Application of ultrasonic tomography to characterize the mechanical state of standing trees ( picea abies )," Journal of Physics: Conference Series, vol. 353, no. 1, p. 012007, March 2012.

[7] A. Guyot, K. T. Ostergaard, M. Lenkopane, J. Fan, and D. A. Lockington, "Using electrical resistivity tomography to differentiate sapwood from heartwood: application to conifers," Tree Physiology, vol. 33, no. 2, pp. 187-194, February 2013.

[8] M. L. Elliott, T. K. Broschat, and L. Göcke, "Preliminary evaluation of electrical resistance tomography for imaging palm trunks," Arboriculture \& Urban Forestry, vol. 42, no. 2, pp. 111-119, March 2016.

[9] J. V. den Bulcke, E. L. Wernersson, M. Dierick, D. V. Loo, B. Masschaele, L. Brabant, M. N. Boone, L. V. Hoorebeke, K. Haneca, A. Brun, C. L. L. Hendriks, and J. V. Acker, "3d tree-ring analysis using helical x-ray tomography,” Dendrochronologia, vol. 32, no. 1, pp. 39 - 46, January 2013.

[10] G. Nicolotti, L. V. Socco, R. Martinis, A. Godio, and L. Sambuelli, "Application and comparison of three tomographic techiques for detection decay in trees," Journal of Arboriculture, vol. 29, no. 2, pp. 66-78, March 2003.

[11] S. A. Al Hagrey, "Geophysical imaging of root-zone, trunk, and moisture heterogeneity," Journal of Experimental Botany, vol. 58, no. 4, pp. 839-854, January 2007.

[12] J. R. Butnor, M. L. Pruyn, D. C. Shaw, M. E. Harmon, A. N. Mucciardi, and M. G. Ryan, "Detecting defects in conifers with ground penetrating radar: Applications and challenges," Forest Pathology, vol. 39, no. 5, pp. 309-322, October 2009.

[13] H. Lorenzo, V. Pérez-Gracia, A. Novo, and J. Armesto, "Forestry applications of ground-penetrating radar," Forest Systems, vol. 19, no. 1, pp. 5-17, April 2010.

[14] L. Fu, S. Liu, and L. Liu, "Internal structure characterization of living tree trunk cross-section using GPR: Numerical examples and field 
data analysis," in Proceedings of the 15th International Conference on Ground Penetrating Radar, GPR 2014, June 2014, pp. 155-160.

[15] E. Mazurek and M. Łyskowski, "Evaluation of gpr surveys for assessment of trees condition in urbanized areas," Geology, Geophysics and Environment, vol. 40, no. 3, pp. 291-296, January 2015.

[16] W. Li, J. Wen, Z. Xiao, and S. Xu, “Application of ground-penetrating radar for detecting internal anomalies in tree trunks with irregular contours," Sensors (Switzerland), vol. 18, no. 2, February 2018.

[17] K. Takahashi and K. Aoike, "Gpr measurements for diagnosing tree trunk," in 2018 17th International Conference on Ground Penetrating Radar (GPR), June 2018, pp. 1-4.

[18] R. J. Ross and F. P. L. USDA Forest Service., "Wood handbook : wood as an engineering material," Tech. Rep., April 2010.

[19] I. Rodríguez-Abad, R. Martínez-Sala, R. Capuz Lladró, R. Díez Barra, and F. García-Garcia, "Assessment of the variation of the moisture content in the pinus pinaster ait using the non destructive gpr technique," Materiales de Construccion, vol. 61, no. 301, pp. 143-156, March 2011.

[20] I. Rodríguez-Abad, R. Martínez-Sala, F. García-García, and R. Capuz-Lladro, "Nondestructive methodologies for the evaluation of moisture content in sawn timber structures: ground-penetrating radar and ultrasound techniques," Near Surface Geophysics, vol. 8, pp. 475-482, December 2010.

[21] N. Pinel, C. Le Bastard, C. Bourlier, and M. Sun, "Asymptotic modeling of coherent scattering from random rough layers: Application to road survey by GPR at nadir," International Journal of Antennas and Propagation, vol. 2012, 2012.

[22] F. Tosti, L. Bianchini Ciampoli, A. Calvi, A. M. Alani, and A. Benedetto, "An investigation into the railway ballast dielectric properties using different GPR antennas and frequency systems," NDT and E International, vol. 93, pp. 131-140, January 2018. 
[23] M. R. M. Ardekani, D. C. Jacques, and S. Lambot, "A layered vegetation model for gpr full-wave inversion," IEEE Journal of Selected Topics in Applied Earth Observations and Remote Sensing, vol. 9, no. 1, pp. 18-28, January 2016.

[24] S. Lambot, M. Antoine, M. Vanclooster, and E. C. Slob, "Effect of soil roughness on the inversion of off-ground monostatic GPR signal for noninvasive quantification of soil properties," Water Resources Research, vol. 42, no. 3, March 2006.

[25] F. Jonard, L. Weihermüller, H. Vereecken, and S. Lambot, "Accounting for soil surface roughness in the inversion of ultrawideband off-ground GPR signal for soil moisture retrieval," GEOPHYSICS, vol. 77, no. 1, pp. H1-H7, January 2012.

[26] A. Giannopoulos, "Modelling ground penetrating radar by gprmax," Construction and Building Materials, vol. 19, no. 10, pp. 755-762, December 2005.

[27] C. Warren, A. Giannopoulos, and I. Giannakis, "gprmax: Open source software to simulate electromagnetic wave propagation for ground penetrating radar," Computer Physics Communications, vol. 209, pp. 163 - 170, December 2016.

[28] J. Ježová, L. Mertens, and S. Lambot, "Ground-penetrating radar for observing tree trunks and other cylindrical objects," Construction and Building Materials, vol. 123, pp. 214-225, October 2016.

[29] J. Ježová, J. Harou, and S. Lambot, "Reflection waveforms occurring in bistatic radar testing of columns and tree trunks," Construction and Building Materials, vol. 174, pp. 388 - 400, June 2018.

[30] A. Taflove, S. C. Hagness, and M. Piket-May, "9 - computational electromagnetics: The finite-difference time-domain method," in The Electrical Engineering Handbook, W.-K. CHEN, Ed. Burlington: Academic Press, 2005, pp. 629 - 670.

[31] J. Ježová and S. Lambot, "Reflection waveforms occurring in gpr tree trunk testing," 2016 16th International Conference on Ground Penetrating Radar (GPR), pp. 1-5, June 2016. 
[32] —, "A dielectric horn antenna and lightweight radar for material inspection," Under Review in Journal of Applied Geophysics, 2018.

[33] S. Lambot, E. C. Slob, I. D. Van Bosch, B. Stockbroeckx, and M. Vanclooster, "Modeling of ground-penetrating radar for accurate characterization of subsurface electric properties," IEEE Transactions on Geoscience and Remote Sensing, vol. 42, no. 11, pp. 2555-2568, November 2004. 
The scientific paper that you have downloaded is included in Issue 1, Volume 2 (March 2019) of the journal Ground Penetrating Radar (ISSN 2533-3100; journal homepage: www.gpradar.eu/journal).

All Ground Penetrating Radar papers are processed and published in true open access, free to both Authors and Readers, thanks to the generous support of TU1208 GPR Association and to the voluntary efforts of the journal Editorial Board. The publication of Issue 1, Volume 2 is also supported by IDS Georadar s.r.1. (idsgeoradar.com). The present information sheet is obviously not part of the scientific paper.
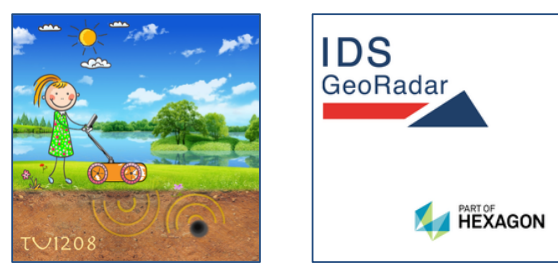